\title{
INSTABILITY DUE TO INTERNAL DAMPING OF ROTATING SHAFTS
}

\author{
W. Lemahieu ${ }^{1}$, M.Loccufier ${ }^{1}$, B. Vervisch ${ }^{1,2}$ and P. De Baets ${ }^{1}$ \\ ${ }^{1}$ Ghent University, Belgium \\ ${ }^{2}$ Howest, Belgium
}

\begin{abstract}
Rotor internal damping has been indicated as one of the main causes of instability in rotating machinery for more than a century. However, the exact characterisation of this damping is still an unsolved research topic. Therefore, in this thesis the consequences of material damping in rotating shafts are examined more in depth. Two main steps are considered. Firstly, a finite element model of the beam, including viscous and hysteretic damping, is constructed. This model allows to calculate the threshold speed of instability and the resonance frequencies of a shaft. Furthermore it allows to vary the damping parameters and to compare the considered models giving an indication of the general relations between instability and damping properties. Secondly, an experimental approach should elucidate which model fits best for the physical damping. In general, the main purpose is to gain new insights into how the damping should really be modelled to have the most accurate and safe prediction of a designed rotor.
\end{abstract}

Keywords: Rotor dynamics; structural damping; stability analysis; threshold speed

\section{INTRODUCTION}

In many modern machinery there is a trend towards increasing the rotating speed because machines operating at higher speed achieve higher performance levels for smaller dimensions. These machines operate typically above the first resonance frequency of the shaft. However, this requires some extra attention from the designer.

Below the first critical speed, the main point of concern is the reduction of the unbalance to limit the vibration forces. Above the first critical speed, also damping forces must be considered to make a safe design. The damping forces can be subdivided into two types [1]. The non-rotating damping (for instance damping of a squeeze film bearing) which is always stabilising and the rotating damping or synchronous damping (e.g. the internal material damping) which tends to be destabilising in supercritical range. In special cases non-synchronous rotating damping can be present [2] (for instance with active bearings or Eddy current dampers). The stabilising of destabilising effect of this kind of damping depends on the rotational speed of the shaft.

Although that damping can cause an unstable threshold speed which cannot be crossed during operation, it is not practically known how many damping is typical for a rotating shaft in practice and how that damping should be modelled. Two widely used damping representations are the viscous damping model and the hysteretic damping model. In this thesis, both of these models are implemented in a finite element model. To find out which of these two models suits the best for the case of a rotating shaft, an experiment is prepared. In that experiment the goal is to estimate the damping in the rotor below and above its first critical speed up to a critical speed. This should give more insights into the behaviour of the damping and allow to predict if instability will occur on an existing machine.

In practice, many other elements may influence the instability of a rotor [3]. The use of journal bearings for example is given as one of the most frequent causes of rotor instability. Joints, fits, groove couplings and coatings are other features that increase the possibility of instability. For this research we try to eliminate these influences to focus on the role of rotor internal damping.

\section{EQUATIONS OF MOTION}

The general equation of motion for static structures (eq. (1)) differs from the equation of motion of rotating structures (eq. (2))

$$
\begin{gathered}
M \ddot{q}+C \dot{q}+K q=f(t) \\
M \ddot{q}+C(\Omega) \dot{q}+K(\Omega) q=f(t)
\end{gathered}
$$

The mass matrix $(M)$ remains symmetric. The stiffness $(K)$ and the damping $(C)$ terms become speed $(\Omega)$ dependent and do not remain symmetric. Both the damping and the stiffness matrix can be split into a sum of a symmetric and a skew-symmetric matrix. 
From the general non-rotating case it is widely known that the symmetric mass matrix and a symmetric stiffness matrix represent conservative elements, while a symmetric damping matrix represent nonconservative elements [4]. In a similar way the effect of the non-symmetric terms of stiffness and damping matrix can be studied. The skew-symmetric part of the damping matrix, is usually called the gyroscopic matrix. It can be proven that this matrix behaves as a conservative system [4], [5]. The skew-symmetric part of the stiffness matrix, called circulatory terms, induces non-conservative terms depending on the speed. For forward whirl motions this induces a positive damping effect while for backward whirls a positive damping is added.

The presence of speed dependent resonance frequencies, forward and backward whirl motions and instability threshold speeds are some of the typical phenomena that are encountered examining rotating systems.

Another important issue is that the formulation of $1^{\text {st }}, 2^{\text {nd }}, 3^{\text {rd }}, \ldots$ mode is not valid in rotating systems. Each static mode splits up in a forward and a backward whirl motion. If an excitation is applied in the same direction of to the rotation direction the forward whirl motion will be induced, the backward whirl motion is induced in the opposite case.

\section{ROLE OF INTERNAL DAMPING AND STABILITY ANALYSIS}

Considering the equation (4), the damping can be split this damping up into several parts: non-rotating damping, rotating damping and a gyroscopic effect (see also equation (4)). The total damping (and thus the stability of the motion) depends clearly on the proportion of these parts.

The influence of rotating and non-rotating damping on the threshold speed of stability can be can be calculated for a simple example as illustrated by Forrai [5]. Assume a rotor (a point mass on a mass less shaft) with viscous internal damping and viscous damped supports. The equations of motion can be written in following form:

$$
\left(M_{t}+M_{R}\right) \ddot{q}+\left(\eta_{V} K_{b}+C_{\text {stat }}-\Omega G\right) \dot{q}+\left(K_{\text {stat }}+K_{b}+\eta_{V} \Omega K_{c}\right) q=0
$$

Using the displacement vector defined as:

$$
q=\left\{\begin{array}{c}
x+i y \\
\theta_{x}+i \theta_{y}
\end{array}\right\}
$$

With $M_{t}$ and $M_{R}$ the translational and the rotational mass matrices, $C_{\text {stat }}$ and $K_{\text {stat }}$ the damping and stiffness matrix of the stationary parts, $\mathrm{K}_{\mathrm{b}}$ and $\mathrm{K}_{\mathrm{c}}$ the bending and the circulatory stiffness matrix, $\mathrm{G}$ the gyroscopical matrix, $\Omega$ the rotational speed and $\eta_{v}$ the viscous internal damping coefficient. These matrices are derived in a publication of Zorzi and Nelson [7].

The introduction of complex coordinates reduces the size of the matrices. The symmetry properties of the real matrices do not remain. Matrices that are symmetric when using the real coordinates approach give hermitian matrices in terms of complex coordinates, whereas skew-symmetric matrices give way to skewhermitian matrices in complex coordinates when using equation (5) as the coordination transformation [5].

When inserting $\mathrm{q}=\mathrm{P} \mathrm{e}^{\lambda t}$, into equation (4) an eigenvalues problem is created with $\lambda$ the eigenvalues and $\mathrm{P}$ the eigenvector:

$$
\left[\left(M_{t}+M_{R}\right) \lambda^{2}+\left(\eta_{V} K_{b}+C_{\text {stat }}-\Omega G\right) \lambda+K_{\text {stat }}+K_{b}+\eta_{V} \Omega K_{c}\right] P=0
$$

For a positive definite $\mathrm{n} \times \mathrm{n}$-matrix $(\mathrm{Z})$ and a $\mathrm{n}$-dimensional vector $(\mathrm{X})$ the following holds:

$$
z=\bar{X}^{T} Z X>0
$$

This converts the matrix into a scalar. Premultiplying equation (5) with $\bar{P}^{T}$, the transverse of the conjugate of the eigenvector, and using the properties of positive definite matrices (equation (7)), equation (6) could be transformed into a scalar equation (8). This implies the introduction of the so called modal mass, stiffness and damping factors indicated by small letters. The conversion of the gyroscopic and circulatory matrices, which are skew-hermitian in the complex coordinates, can also be converted into a scalar by using an alternative formulation of these two matrices: $\mathrm{G}=i \mathrm{G}^{\prime}$ and $\mathrm{K}_{\mathrm{c}}=-\mathrm{K}_{\mathrm{b}}{ }^{\prime}$ [7]. The matrices $\mathrm{G}^{\prime}$ and $\mathrm{K}_{\mathrm{c}}{ }^{\prime}$ are now hermitian and can also be converted into a positive scalar.

$$
m \lambda^{2}+\left(\eta_{V} k_{b}+c_{\text {stat }}-i \Omega g\right) \lambda+k_{\text {stat }}+k_{b}-i \eta_{V} \Omega k_{b}=0
$$


To determine the threshold speed for instability an eigenvalue with a real part equal to zero is substituted:

$$
-m \omega^{2}+\left(\eta_{V} k_{b}+c_{\text {stat }}-i \Omega g\right) \omega+k_{\text {stat }}+k_{b}-i \eta_{V} \Omega k_{b}=0
$$

Separating the imaginary and the real parts of equation (9) leads to two equations which allow to calculate the threshold instability speed as:

$$
\Omega=\sqrt{\frac{k}{m}}\left(1+\frac{c_{s t a t}}{\eta_{V} k_{b}}\right)
$$

Genta [8] derived the same result but by studying a Jeffcott rotor (a point mass attached to the middle of a massless shaft) supported by viscous dampers. In a similar way, also hysteretic damping can be introduced into the equation of motion as shown by Forrai [9].

Equation (9) shows the influence of the stationary damping (damping of the bearings for example) relative to the internal damping on the rotational threshold speed. This results in the following important conclusions:

- Non-rotational damping will always enlarge the instability threshold speed.

- Backward whirling modes are always stable

- Instability due to rotor internal damping can only occur in supercritical speeds.

\section{FINITE ELEMENT MODEL}

In the previous paragraph it is shown that the instability threshold speed changes with the damping model and the amount of rotational and non-rotational damping. To investigate this more in practical matter, a finite element model was constructed in Matlab®.

Figure 1 gives an overview of the structure of the program. The dimensions and properties of the shaft and bearings are required as inputs for the system. Subsequently the mass, stiffness en damping matrices are constructed using the matrices derived by Zorzi and Nelson [7]. With these matrices the eigenvalues problem is solved for every rotation speed. The resulting eigenvalues contain in the imaginary part the resonance frequencies and in the real part the damping or stability. As a result of the model the resonance frequencies are obtained and decay rate in function of the rotational speed, the so called Campbell diagram and decay rate plot, which allows determining the threshold speed for instability.

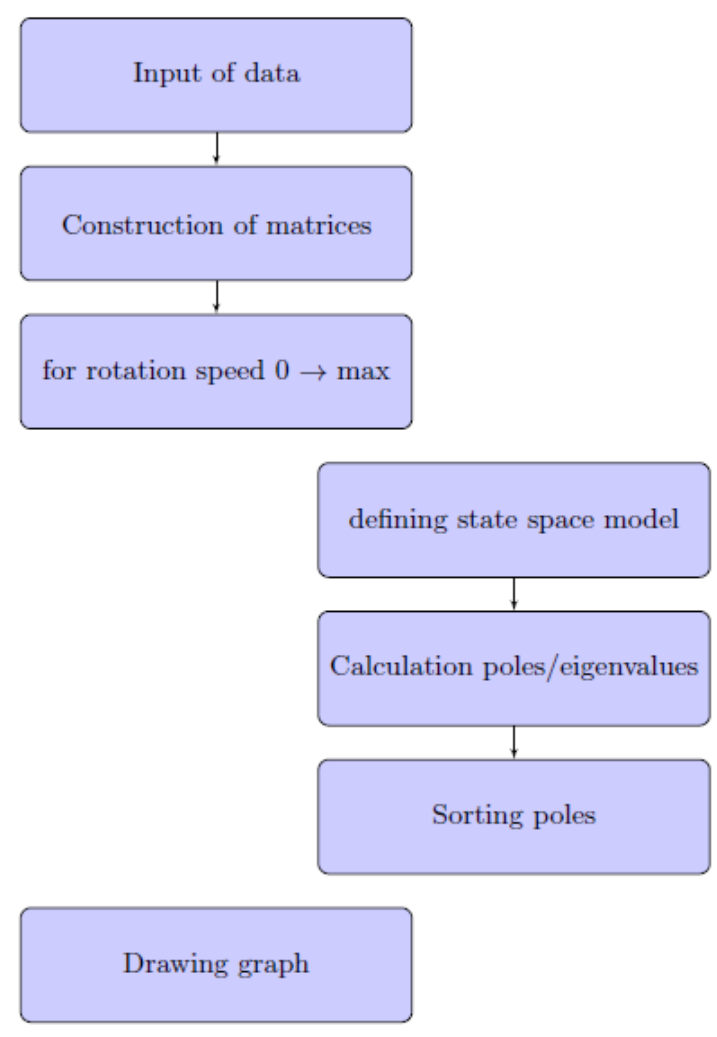

Figure 1. Block diagram of the finite element simulation program. 
The simulation is designed for cylindrical shafts on symmetric bearings. For the bearing damping only a viscous damping coefficient is used while for the internal damping of the rotor a viscous and a hysteretic model is implemented. The model was verified using [7] and [10]. As an example the results for a shaft are demonstrated using following data: $L=1.27 \mathrm{~m}, D=0.1016 \mathrm{~m}, E=208^{*} 10^{9} \mathrm{~N} / \mathrm{m}, \rho=7830 \mathrm{~kg} / \mathrm{m}^{3}, C_{\text {bearing }}=1750$ $\mathrm{Ns} / \mathrm{m}, \mathrm{K}_{\text {bearing }}=1.75^{\star} 10^{7} \mathrm{~N} / \mathrm{m}$.

Figure 2 clearly shows the difference between the two damping models. Viscous damping causes a as well as proportional variation of the decay rate while hysteretic damping induces a constant decay rate with a step at the resonance frequency in the forward modes.

When both models are compared, we can conclude that in the proximity of a resonance frequency, or thus where the frequency of the internal bending motion tends to zero, the hysteretic damping model gives inconsistent results. On the other hand, in the areas further away from this frequency the viscous damping model will induce incorrect results because it is known that at higher deformation frequencies metals behave different than liquids and thus the viscous damping model will not satisfy.
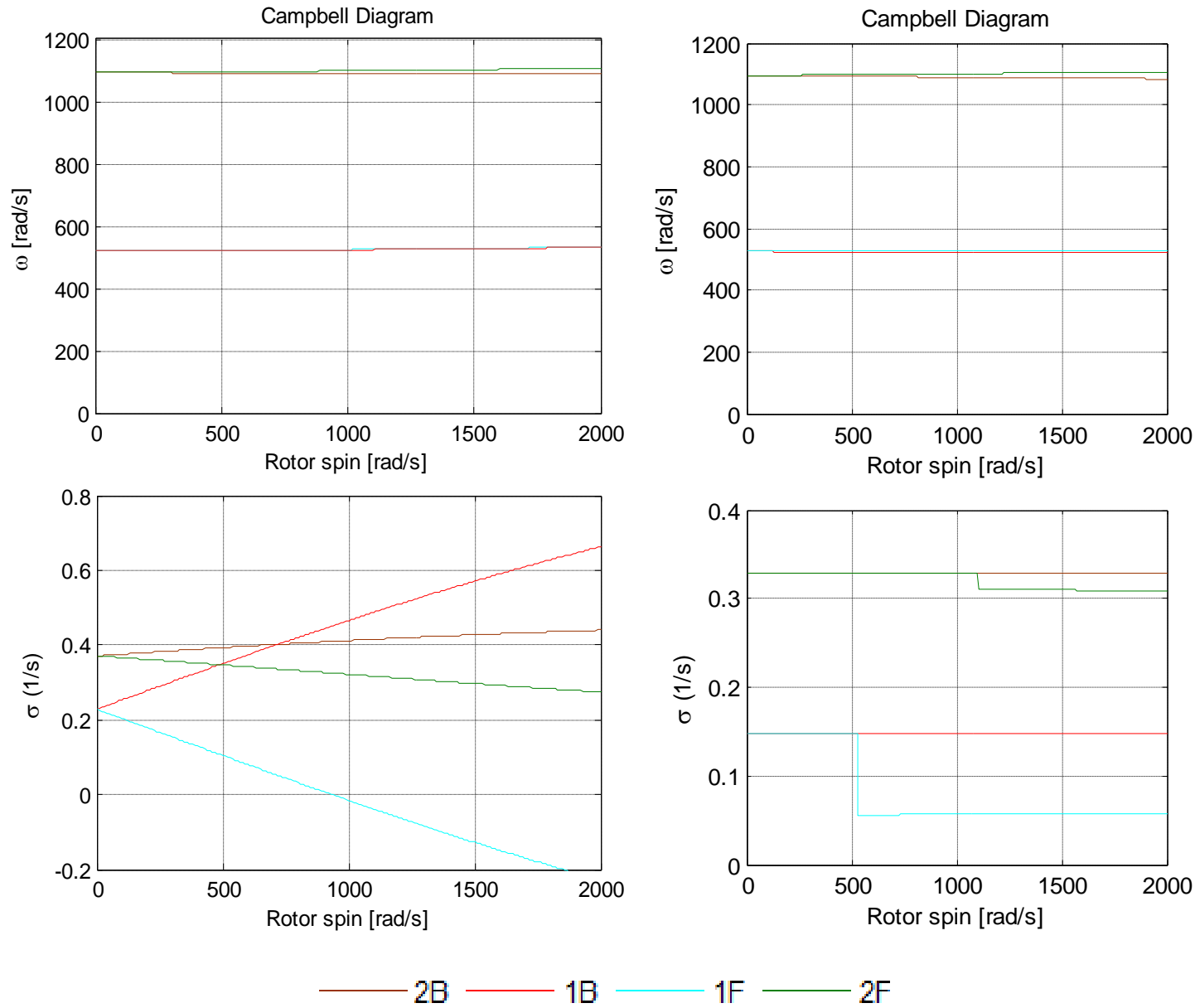

Figure 2. First and second forward and backward whirl mode logarithmic decrement and Campbell diagram for (a) a viscous damped rotor with $\eta_{V}=0.002$ (b) a hysteretic damped rotor with $\eta_{H}=0.04$.

\section{EXPERIMENTAL APPROACH}

The next step in this thesis is experiments for measuring the practical damping and threshold speeds of a shaft. We start with tests on a shaft simply supported by two wires to investigate the optimum measurement techniques. Subsequently, tests on the stationary shaft mounted in its bearings and finally measurements on the rotating shaft. To ensure that data obtained in each test are comparable, the testing method must be repeatable for every experiment. This last requirement and the fact that we are dealing with a rotating shaft complicates the choice for excitation and measurement techniques.

Bucher and Ewins [11] give an overview of the advantages and disadvantages of some possible excitation techniques for rotating structures that can be used. It can be concluded that several ways are possible but one should be very aware of the possible fault that can be introduced by the rotational movements. 
Hammer impact appears to be the most promising excitation technique for this purpose because this technique affects only slightly the properties of the structure. For the measurement of the vibration response the generally used accelerometers are no longer useful. Laser meters or eddy current probes are more designated for this purpose.

By performing the same measurement with different rotational speeds the variation of damping could be compared to the theoretical finite element models.

\section{CONCLUSIONS}

This research investigates the link between the theoretical models for internal damping in rotating structures and the practical observations. In the analytical part of this thesis a finite element model was designed that is capable of determine the damping, resonance frequencies and instability of the shaft. On the practical side, a test rig was used to perform vibration measurements. Combining these two elements leads to a more profound insight into the actual damping phenomena and to a more realistic way to predict the instability constraints and the safe operating zones of a rotor.

\section{NOMENCLATURE}

C Damping matrix

$\mathrm{C}_{\text {stat }}$ Stationary damping matrix

G Gyroscopic matrix

K Stiffness matrix

$\mathrm{K}_{\mathrm{b}} \quad$ Bending stiffness matrix

$\mathrm{K}_{\mathrm{c}} \quad$ Circulatory stiffness matrix

$\mathrm{K}_{\text {stat }}$ Stationary stiffness matrix
M Mass matrix

$M_{R} \quad$ Rotational mass matrix

$M_{T} \quad$ Transverse mass matrix

q Displacement vector

$\Omega \quad$ Rotation frequency $\quad \mathrm{rad} / \mathrm{s}$

$\omega \quad$ Whirl frequency $\quad \mathrm{rad} / \mathrm{s}$

$\eta_{\mathrm{V}} \quad$ Viscous damping coefficient

$\eta_{H} \quad$ Hysteretic damping coefficient

\section{REFERENCES}

[1] Genta, G., On a Persistent Misunderstanding of the Role of Hysteretic Damping in Rotordynamics, Journal of Vibration and Acoustics, 126, 3, 459, 2004.

[2] Genta, G., On the Role of Nonsynchronous Rotating Damping in Rotordynamics, International Journal of Rotating Machinery, 6, 6, 467-475, 2010.

[3] Kandil, M.A., On rotor internal damping instability, 256, 2004.

[4] Adams, M., Insights into linearized rotor dynamics, Journal of Sound and Vibration, 76, 129-142, 1981.

[5] Adams, M., Insights into linearized rotor dynamics, Part 2, Journal of Sound and Vibration, 112, 97-110, 1987.

[6] Forrai, L., A finite element model for stability analysis of symmetrical rotor systems with internal damping, JCAM, 1, 37-47, 2000.

[7] Zorzi, E.S., Nelson, H.D., Finite Element Simulation of Rotor-Bearing Systems With Internal Damping, Journal of Engineering for Power, 99, 71-76, 1977.

[8] Genta, G., Dynamics of rotating systems, Springer Verlag, 2005.

[9] Forrai, L., Instability due to internal damping of symmetrical rotor-bearing systems, JCAM, 1, 137-147, 2000.

[10]Montagner, O., Hochard, C., Dynamic instability of supercritical driveshaft's mounted on dissipative supports: Effects of viscous and hysteretic internal damping, Journal of Sound and Vibration, vol. 305, 3, 378-400, 2007.

[11]Bucher, I., Ewins, D.J., Modal analysis and testing of rotating structures, Philosophical Transactions of the Royal Society A: Mathematical, Physical and Engineering Sciences, 359, 61-96, 2001. 